\title{
COMMD5 Inhibits Malignant Behavior of Renal Cancer Cells
}

\author{
JIN IKEDA ${ }^{1}$, HIROYUKI MATSUDA ${ }^{2}$, MAIKO OGASAWARA ${ }^{1}$, YUKIMOTO ISHII ${ }^{2}$, \\ KENYA YAMAGUCHI $^{3}$, SATORU TAKAHASHI ${ }^{3}$, NOBORU FUKUDA ${ }^{4}$, YOSHIKAZU MASUHIRO ${ }^{5}$, \\ MORITO ENDO ${ }^{6}$, MASAYOSHI SOMA $^{1}$, PAVEL HAMET $^{7,8}$ and JOHANNE TREMBLAY 7,8 \\ ${ }^{1}$ Division of General Medicine, Department of Internal Medicine, \\ Nihon University School of Medicine, Tokyo, Japan; \\ ${ }^{2}$ Division of Medical Research Planning and Development, Nihon University School of Medicine, Tokyo, Japan; \\ ${ }^{3}$ Department of Urology, Nihon University School of Medicine, Tokyo, Japan; \\ ${ }^{4}$ Division of Cell Regeneration and Transplantation, Department of Functional Morphology, \\ Nihon University School of Medicine, Tokyo, Japan; \\ ${ }^{5}$ Department of Applied Biological Sciences, College of Bioresource Sciences, Nihon University, Fujisawa, Japan; \\ ${ }^{6}$ Faculty of Human Health Science, Hachinohe Gakuin University, Aomori, Japan; \\ ${ }^{7}$ Centre de Recherche, Centre Hospitalier de l'Université de Montréal (CRCHUM), Montréal, QC, Canada; \\ ${ }^{8}$ Department of Medicine, Université de Montréal, Montréal, QC, Canada
}

\begin{abstract}
Background/Aim: Copper metabolism MURRI domain-containing 5 (COMMD5) is mainly expressed in renal tubules (RTs), where it facilitates re-differentiation of injured $R T s$. We reported that COMMD5 regulates the expression of epidermal growth factor receptor by participating in its endocytic membrane trafficking, thus inhibiting tumor growth. Here we aimed to determine the role of COMMD5 in malignant phenotypes of renal cell carcinoma (RCC). Materials and Methods: The associations between COMMD5 levels in RTs adjacent to RCC tumors in patients and their clinicopathologic characteristics were evaluated, and the effects of COMMD5 on cancer stemness in RCC cells were investigated. Results: Low COMMD5 levels in RTs correlated with high tumorigenesis and poor patient outcomes. COMMD5 overexpression in RCC cells reduced the proportion of cancer stem cell-like cells and their malignant phenotypes, including proliferation, invasion and sphere formation. Secreted COMMD5 from RT cells also reduced malignant phenotypes. Conclusion: COMMD5 might suppress malignant phenotypes of RCC, thus inhibiting tumor development and improving patient prognosis.
\end{abstract}

This article is freely accessible online.

Correspondence to: Hiroyuki Matsuda, Division of Medical Research Planning and Development, Nihon University School of Medicine, 30-1 Ooyaguchi-Kamicho, Itabashi-ku, Tokyo 173-8610, Japan. Tel: +81 339728111, Fax: +81 339728666, e-mail: matsuda.hiroyuki75@nihon-u.ac.jp

Key Words: COMMD5, renal cell carcinoma, renal tubules, cancer stem cell, metastasis and recurrence.
Copper metabolism MURR1 domain-containing 5 (COMMD5), also known as hypertension-related, calcium-regulated gene, is characterized by a conserved COMM domain at its carboxyterminal end (1). Its expression is higher in the parathyroid and kidney of spontaneously hypertensive rats than in normotensive rats (2). Functional studies indicated that COMMD5 overexpression in kidney cells facilitates their differentiation, with a lower proliferative capacity (3). Overexpression of COMMD5 in proximal renal tubules (RTs) of mice accelerated tubular repair after acute kidney injury by facilitating redifferentiation of injured RT cells through the induction of $\mathrm{P} 21^{\mathrm{CIP} 1 / \mathrm{WAF} 1}$ via a p53-independent pathway, resulting in faster recovery of renal function and a superior survival rate (4).

Cancer afflicted about 18.1 million new patients worldwide in 2018 and accounted for nearly 9.6 million related deaths (5). Cancer morbidity and mortality are rapidly growing in every region of the world. The high mortality rate often correlates with a lack of clear symptoms, which results in late diagnosis for patients and the development of cancer resistant to chemotherapy and radiotherapy. Recent progress in cancer research has revealed that the presence of cancer stem cells (CSCs) is pivotal for the development of malignant phenotypes of various cancer types, including invasion, metastasis, recurrence and resistance to therapy (6-8). CSCs are small populations of cells that have the potential to reproduce and sustain cancer and the abilities of multiple differentiation and self-renewal $(9,10)$.

Malignant kidney cancers afflict about 350,000 new patients worldwide, and the incidence of renal cell carcinoma (RCC) is increasing annually $(11,12)$. Advanced kidney cancer in adults is resistant to conventional chemotherapy 
and radiotherapy (13). In recent years, CSC-like cells have been identified in human RCC cell lines and renal carcinoma specimens (14). These cells are characterized by rapid proliferation, self-renewal capability, multiple differentiation, resistance to chemotherapy and radiotherapy, and tumor formation ability (15). We initially showed that COMMD5 levels were lower in different cancer cell lines from glioblastoma, a partly differentiated RCC and a moderately differentiated hepatocellular tumor, than in the corresponding normal cells (2). In addition, COMMD5 overexpression in murine renal cancer cells inhibited their proliferation and in vivo tumor formation (16). Moreover, COMMD5 is frequently underexpressed, not only in specimens of human clear-cell RCC (ccRCC) but also in non-tumorous tubules adjacent to ccRCCs of large size in patients.

We hypothesized that a low level of COMMD5 expression in the kidneys would contribute to the transformation of precancerous cells into CSCs and augment malignant phenotypes in RCC. In the present study, we examined the associations between COMMD5 expression level in the kidneys and clinicopathological characteristics of ccRCC. We next investigated the effects of COMMD5 on CSCs in a human RCC cell line. This study allowed us to determine the expression and clinical relevance of COMMD5 in patients with RCC.

\section{Materials and Methods}

Patients and samples. This study received the approval of the Examination Committee for Clinical Research of Nihon University Itabashi Hospital (RK-131213-9). Patients with ccRCC who underwent surgery at Nihon University Itabashi Hospital (Tokyo, Japan) from 1997 to 2006 were enrolled. All patients provided written informed consent prior to surgery. The samples were collected from the archives of the Department of Pathology of Nihon University. Clinical characteristics, such as age, sex and serum creatinine level, were obtained from the electronic records. Tumor size was based on the longest diameter of pathological specimens. Kaplan-Meier curves were generated for determining overall, metastasis- and recurrence-free survival, defined as the interval between primary resection and death from disease, disease metastasis and recurrence or the last follow-up date, respectively, were compared using the log-rank test.

For immunohistochemical staining of COMMD5, tissue sections including ccRCC and renal cortex were incubated with antiCOMMD5 (Proteintech Group Inc., Chicago, IL, USA) overnight as described previously (4), followed by visualization using a Cell and Tissue Staining kit (R\&D Systems, Minneapolis, MN, USA) in accordance with the manufacturer's recommendations. Finally, sections were counterstained with Mayer's hematoxylin. All slides were reviewed by a pathologist blinded to the experimental groups. The COMMD5 staining index of RTs was calculated as the product of the staining intensity multiplied by the percentage of positively stained area, producing an index ranging from 1 to 4 . Patients were then divided into groups with low (index 1 of and 2) and high (index of 3 and 4) COMMD5 expression.
Stable transfection and cell culture. Human RCC23 cells were a gift from Dr. Kyoko Fujiwara (Nihon University School of Dentistry, Tokyo, Japan); HK-2, RT epithelial cell line from normal adult human kidney, and TCMK-1 mouse kidney epithelial cells were obtained from the American Type Culture Collection (Rockville, MD, USA). Both the RCC23 and TCMK-1 cell lines were transfected with control plasmid (pcDNA/Neo1; Thermo Fisher Scientific, Waltham, MA, USA) or plasmid encoding rat COMMD5 using Attractene transfection reagent (Qiagen, Valencia, CA, USA) following the manufacturer's protocol (16). Transfected cells were first selected in medium containing G418 (Merck Millipore, Billerica, MA, USA). After isolation of single clones, the cells were grown in Roswell Park Memorial Institute (RPMI)-1640 medium (Sigma-Aldrich, St. Louis, MO, USA) or Dulbecco's modified Eagle's medium/nutrient mixture F-12 (Sigma-Aldrich).

siRNA knockdown. Commd5 was knocked down with small interfering RNA (siRNA) targeting a human COMMD5 sequence (siCOMMD5; Thermo Fisher Scientific). Wild-type (WT)-RCC23 cells and WT-HK-2 cells were transfected with $25 \mathrm{nM}$ of non-target control siRNA (siNC; Thermo Fisher Scientific) or siCOMMD5 with HiPerFect transfection reagent (Qiagen), according to the manufacturer's instructions. At $48 \mathrm{~h}$ after transfection, the cells were harvested, and knockdown efficacy was assessed by real-time polymerase chain reaction (PCR) and western blot.

mRNA quantification and expression. Total RNA was isolated from RCC23 cells stably transfected with control plasmid (Neo-RCC23) or an expression plasmid containing rat Commd5 cDNA (COMMD5RCC23), and WT-RCC23 cells transfected with siCOMMD5 (siCOMMD5-RCC23) or siNC (siNC-RCC23) with TRIzol reagent (Thermo Fisher Scientific), according to the manufacturer's instructions. Isolated total RNA was reverse-transcribed as described previously (4). Real-time quantitative PCR was performed with diluted cDNA using designed TaqMan probes (Thermo Fisher Scientific) according to the manufacturer's instructions. Real-time PCR data were analyzed with standard curves and normalized to those of glyceraldehyde-3-phosphate dehydrogenase with its specific primer sets (5' and 3' primers: 5'-TGCACCACCAACTGCTTAGC3' and 5'-GGCATGGACTGTGGTCATGAG-3') as described previously $(4,17)$. Correlation coefficients for each standard curves all exceeded 0.95 .

Western blot. Cultured cells and acetone-precipitated supernatants were lysed in a modified radioimmunoprecipitation assay protein extraction buffer as described previously (4). Each sample was applied equally to $12 \%$ polyacrylamide gels and transblotted to polyvinylidene difluoride membranes (GE Healthcare, Uppsala, Sweden). After blocking, samples were incubated with primary antibodies to $\beta$-actin, glyceraldehyde 3-phosphate dehydrogenase (Santa Cruz Biotechnology, Inc., Santa Cruz, CA, USA) and COMMD5 overnight, followed by incubation with secondary horseradish peroxidase-conjugated antibodies (Santa Cruz Biotechnology, Inc.). Immunocomplexes were visualized by the western blot and chemiluminescence imaging system FUSION Solo $\mathrm{S}$ (VILBER, Collégien, France).

Cell proliferation assay. Neo- or COMMD5-RCC23 cells, and siNC- or siCOMMD5-RCC23 cells were seeded onto 96-well microliter plates. After $24 \mathrm{~h}$ of serum starvation, cell proliferation 
Table I. Multivariate logistic analysis of factors associated with large tumor size $(>40 \mathrm{~mm})$.

\begin{tabular}{lcll}
\hline Variable & No. of cases & OR (95\% CI) & $p$-Value \\
\hline Age & & & \\
$\quad>50$ Years & 98 & $0.66(0.21-2.04)$ & 0.47 \\
$\quad \leq 50$ Years & 19 & 1 & \\
Sex & 34 & $3.08(1.19-7.95)$ & $\mathbf{0 . 0 2}$ \\
$\quad$ Female & 83 & 1 & \\
$\quad$ Male & & & \\
Creatinine level & 25 & $2.41(0.87-6.71)$ & 0.091 \\
$\quad$ Abnormal & 92 & & \\
$\quad$ Normal & & & \\
Fuhrman grade & 10 & $10.6(1.18-95.2)$ & $\mathbf{0 . 0 3 5}$ \\
$>2$ & 107 & 1 & \\
$\quad \leq 2$ & 65 & $2.8(1.22-6.44)$ & $\mathbf{0 . 0 2}$ \\
COMMD5 in normal RTs & & 1 & \\
$\quad$ Low & 52 & & \\
High & &
\end{tabular}

CI: Confidence interval; COMMD5: copper metabolism MURR1 domain-containing 5; OR: odds ratio; RTs: renal tubules. Significant $p$ Values are shown in bold.

was measured with PrestoBlue Cell Viability Reagent (Thermo Fisher Scientific) according to the manufacturer's recommendations. The fluorescent signal was measured (excitation $530 \mathrm{~nm}$ and emission $570 \mathrm{~nm}$ ) using a Multilabel counter 1420 Victor $^{3} \mathrm{~V}$ (Perkin Elmer, Wellesley, MA, USA).

Cell invasion assay. The effect of COMMD5 on tumor cell invasiveness was assessed by measuring cell invasion with a CytoSelect ${ }^{\mathrm{TM}}$ 96-well cell invasion assay kit (Cell Biolabs, Inc., San Diego, CA, USA) in accordance with the manufacturer's instructions. Briefly, a suspension of $15 \times 10^{5} \mathrm{NeO}-$ and COMMD5RCC23 cells in $100 \mu \mathrm{l}$ of serum-free RPMI medium or a suspension of $15 \times 10^{5}$ WT-RCC23 in $100 \mu \mathrm{l}$ of cell-culture supernatants from Neo- and COMMD5-TCMK-1 cells were placed on top of the basement membrane matrix inside the upper chamber and were allowed to invade towards $10 \%$ fetal bovine serum through the matrix and adhere to the bottom membrane of the insert. After 24 $\mathrm{h}$, non-invading cells were removed from the upper chamber, and invading cells on the bottom of the invasion membrane were lysed and quantified using CyQuant ${ }^{\circledR}$ GR fluorescent dye in a microplate reader at a wavelength of $480 \mathrm{~nm}$.

Sphere-formation assay. Neo- and COMMD5-RCC23 cells, or WTRCC23 cells were suspended and incubated in serum-free RPMI1640 medium or serum-free cell-culture supernatants from Neo- and COMMD5-TCMK-1 cells supplemented with $10 \mathrm{ng} / \mathrm{ml}$ of basic fibroblast growth factor (Sigma-Aldrich) and epidermal growth factor (Sigma-Aldrich) at a cell density of $1.5 \times 10^{4}$ cells per well in ultralow-attachment 6-well plates (Greiner Bio-One International $\mathrm{GmbH}$, Kremsmunster, Austria). WT-RCC23 cells were harvested $48 \mathrm{~h}$ after transfection of siNC or siCOMMD5 then the cells were seeded at a cell density of $1.5 \times 10^{4}$ cells per well onto 6 -well plates. After 14 days, cell spheres of diameter greater than $500 \mu \mathrm{m}$ in each well were counted using a microscope (Olympus IX73; Olympus, Tokyo, Japan) directly on the plate.
Table II. Univariate Cox proportional-hazards analysis of factors associated with metastasis and recurrence.

\begin{tabular}{lll}
\hline Variable & HR $(95 \% \mathrm{CI})$ & $p$-Value \\
\hline Age & & \\
$\quad>50$ Years & $0.69(0.28-1.69)$ & 0.412 \\
$\quad \leq 50$ Years & 1 & \\
Sex & & \\
$\quad$ Female & $2.21(1.06-4.60)$ & $\mathbf{0 . 0 3 3}$ \\
$\quad$ Male & 1 & \\
Creatinine level & $1.23(0.53-2.88)$ & 0.634 \\
$\quad$ Abnormal & 1 & \\
$\quad$ Normal & & \\
Fuhrman grade & $2.53(0.96-6.63)$ & 0.06 \\
$\quad>2$ & 1 & \\
$\quad \leq 2$ & $10.25(3.10-33.92)$ & $<\mathbf{0 . 0 0 0 1}$ \\
Tumor size & 1 & \\
$\quad>40$ mm & & $\mathbf{0 . 0 4 6}$ \\
$\quad \leq 40$ mm & $2.29(1.01-5.18)$ & \\
COMMD5 in normal RTs & 1 & \\
$\quad$ Low & &
\end{tabular}

CI: Confidence interval; COMMD5: copper metabolism MURR1 domain-containing 5; HR: hazard ratio; RTs: renal tubules. Significant $p$-Values are shown in bold.

Analysis of aldehyde dehydrogenase (ALDH) activity. An ALDEFLUOR $^{\mathrm{TM}}$ Kit (STEMCELL Technologies Inc., Vancouver, $\mathrm{BC}$, Canada) was used to detect cells expressing high levels of ALDH (18). In brief, $1.0 \times 10^{6} \mathrm{Neo}-$ and COMMD5-RCC23 cells were resuspended in $1 \mathrm{ml}$ of ALDEFLUOR ${ }^{\mathrm{TM}}$ assay buffer containing $5 \mu \mathrm{l}$ of activated ALDEFLUOR ${ }^{\mathrm{TM}}$ reagent. As a negative control for each sample of cells, $500 \mu \mathrm{l}$ of the cell suspension was immediately transferred to another tube supplemented with $5 \mu \mathrm{l}$ of ALDEFLUOR $^{\mathrm{TM}}$ diethylaminobenzaldehyde reagent, a potent inhibitor of cytosolic ALDH1. After incubation at $37^{\circ} \mathrm{C}$ for $30 \mathrm{~min}$, ALDH-positive Neo- and COMMD5-RCC23 cells were analyzed by flow cytometry.

Statistical analysis. Values are reported as mean \pm standard error of the mean. Values and percentages between groups were analyzed by Student $t$-test, multiple logistic regression analysis or univariate Cox proportional-hazards analysis. A value of $p<0.05$ was considered to be statistically significant. All analyses were performed using JMP software v14 (SAS Institute Inc., Cary, NC, USA).

\section{Results}

Low COMMD5 expression is associated with ccRCC progression. Tumor size at the time of surgery is one of the prognostic predictors of ccRCC, hence we first divided patients into two groups according to tumor size $(\leq 40 \mathrm{~mm}$ and $>40 \mathrm{~mm}$ ) and the correlation of tumor size with survival was analyzed. The data regarding 117 patients with ccRCCs are shown Table I. Figure 1A illustrates the metastasis- and recurrence-free survival curves during 5 years of follow-up according to tumor size. A ccRCC tumor size larger than 40 
mm was significantly $(p<0.0001)$ associated with worse clinical outcome.

Next, we immunohistochemically examined COMMD5 expression in human ccRCCs and adjacent non-neoplastic kidney tissue from 117 patients. Patients were then divided into two groups according to the intensity of COMMD5 staining in RTs adjacent to ccRCC. In the group with high COMMD5 expression, COMMD5 expression was much lower in ccRCCs than in their paired RTs (Figure 1B). The mean tumor diameter was significantly $(p=0.0205)$ smaller when COMMD5 expression level was high in adjacent nonneoplastic kidney tissue than when COMMD5 expression level was low $(42.9 \pm 3.2 \mathrm{~mm}$ and $54.0 \pm 3.3 \mathrm{~mm}$, respectively) (16). In addition, multivariate analysis indicated that COMMD5 expression in adjacent non-neoplastic kidney tissue was related to an increased risk of large tumor size (Table I).

In the group with low COMMD5 expression, 21 out of 65 patients were diagnosed as having tumor metastasis or recurrence, whereas in that with high COMMD5 expression, only eight out of 52 patients developed tumor metastasis or recurrence within 5 years after surgery. As the number of cases in this study was small, we used a univariate Cox proportional-hazards model to estimate the relative hazard of the metastasis- and recurrence-associated prognostic factors. In this univariate analysis, three factors were significantly related to tumor metastasis and recurrence: Sex, tumor size and COMMD5 expression in normal RTs (Table II). In addition, a Kaplan-Meier plot showed that a low COMMD5 level in normal RTs was significantly $(p=0.0389)$ associated with poor metastasis- and recurrence-free survival (Figure 1A) (16). Thus, low COMMD5 expression in normal RTs might be a risk factor for tumor growth, metastasis and recurrence in ccRCC.

COMMD5 inhibits tumor cell proliferation. To test whether COMMD5 inhibits tumor growth of ccRCCs, we first evaluated the impact of its overexpression in human RCC23 cells. The cells were stably transfected with control plasmid (Neo-RCC23) or an expression plasmid containing rat Commd5 cDNA (COMMD5-RCC23) as described previously (16). As expected, exogenous expression of rat Commd5 mRNA was detected only in the COMMD5-RCC23 cells, whereas endogenous expression of human COMMD5 mRNA was not different between the Neo- and COMMD5-RCC23 cells (Figure 2A). The total COMMD5 protein level was higher in stably transfected COMMD5-RCC23 cells than in Neo-RCC23 cells (Figure 2B). COMMD5 overexpression significantly inhibited proliferation of RCC23 cells (Figure 2C) as we previously reported for other cancer cell types (16).

We then tested the effect of loss of COMMD5 expression on the growth of RCC cells by using specific siRNA. The mRNA and protein levels of COMMD5 were drastically reduced after treatment with siCOMMD5, indicating its successful gene silencing (Figure 3A and B). Loss of COMMD5 expression by siRNA resulted in a significant increase of cell proliferation in RCC23 cells after $24 \mathrm{~h}$ compared with the cells treated with non-targeted control siRNA (Figure 3C).

COMMD5 inhibits cancer stemness. To explore the possibility that COMMD5 might reduce the malignant phenotype of RCC cells, we performed a sphere colonyformation assay, which is commonly used to identify CSCs (19). As shown in Figure 4A, overexpression of COMMD5 significantly reduced the number of cell spheres compared with Neo-control cells 14 days after culture, while loss of COMMD5 expression enhanced the spheroid production of RCC23 cells (Figure 4B).

ALDHs are enzymes that participate in cellular homeostasis through the reaction of aldehyde detoxification. Recent evidence suggests that high ALDH1 enzyme activity as measured by the ALDEFLUOR assay is associated with increased drug resistance, metastasis and self-renewal of CSCs in various cancer types, including RCC $(14,20)$. A shift to the right of the fluorogram, indicative of an ALDH-positive population, was detected by ALDEFLUOR assay in NeoRCC23 cells but not in COMMD5-RCC23 cells (Figure 4C).

To assess the impact of COMMD5 on tumor cell invasiveness, a suspension of Neo- and COMMD5-RCC23 cells was deposited on top of a basement membrane matrix. After $24 \mathrm{~h}$, cells that had invaded through the matrix were lysed and quantified, showing that COMMD5 overexpression significantly inhibited tumor cell invasion (Figure 4D). Overall, our results demonstrate that COMMD5 suppressed the cancer-related phenotype of RCC cells.

Secretory COMMD5 inhibits cancer stemness. We previously reported that COMMD5 protein is secreted from cells into the culture medium (16). Here, we show that secretion of COMMD5 protein was higher in the cell-culture supernatant of COMMD5-overexpressing mouse kidney epithelial TCMK-1 (COMMD5-TCMK-1) cells than that of control Neo-TCMK-1 cells (Figure 5A, left panel). Human kidney epithelial HK-2 cells also secreted endogenous COMMD5 in the culture medium, and levels of both intracellular and secreted COMMD5 protein were reduced by siCOMMD5 in HK-2 cells (Figure 5A, right panel).

The culture medium of HK-2 cells under the treatment of siCOMMD5 might contain siRNA against human COMMD5, hence cell-culture supernatant of COMMD5TCMK-1 or Neo-TCMK-1 cells was used to avoid the effect of loss of COMMD5 by siCOMMD5 on WT-RCC23 cells in the subsequent experiments. The cell-culture supernatant of COMMD5-TCMK-1 cells (containing more COMMD5) inhibited proliferation of WT-RCC23 cells compared with cells incubated with cell-culture supernatant of Neo-TCMK- 

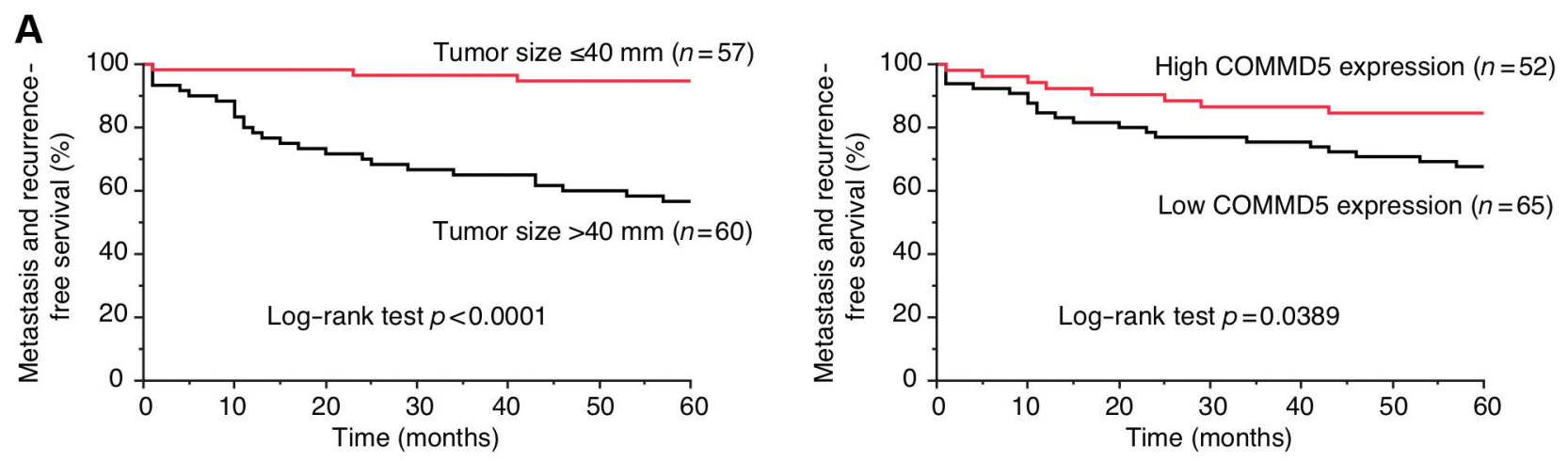

\section{B High COMMD5 expression}
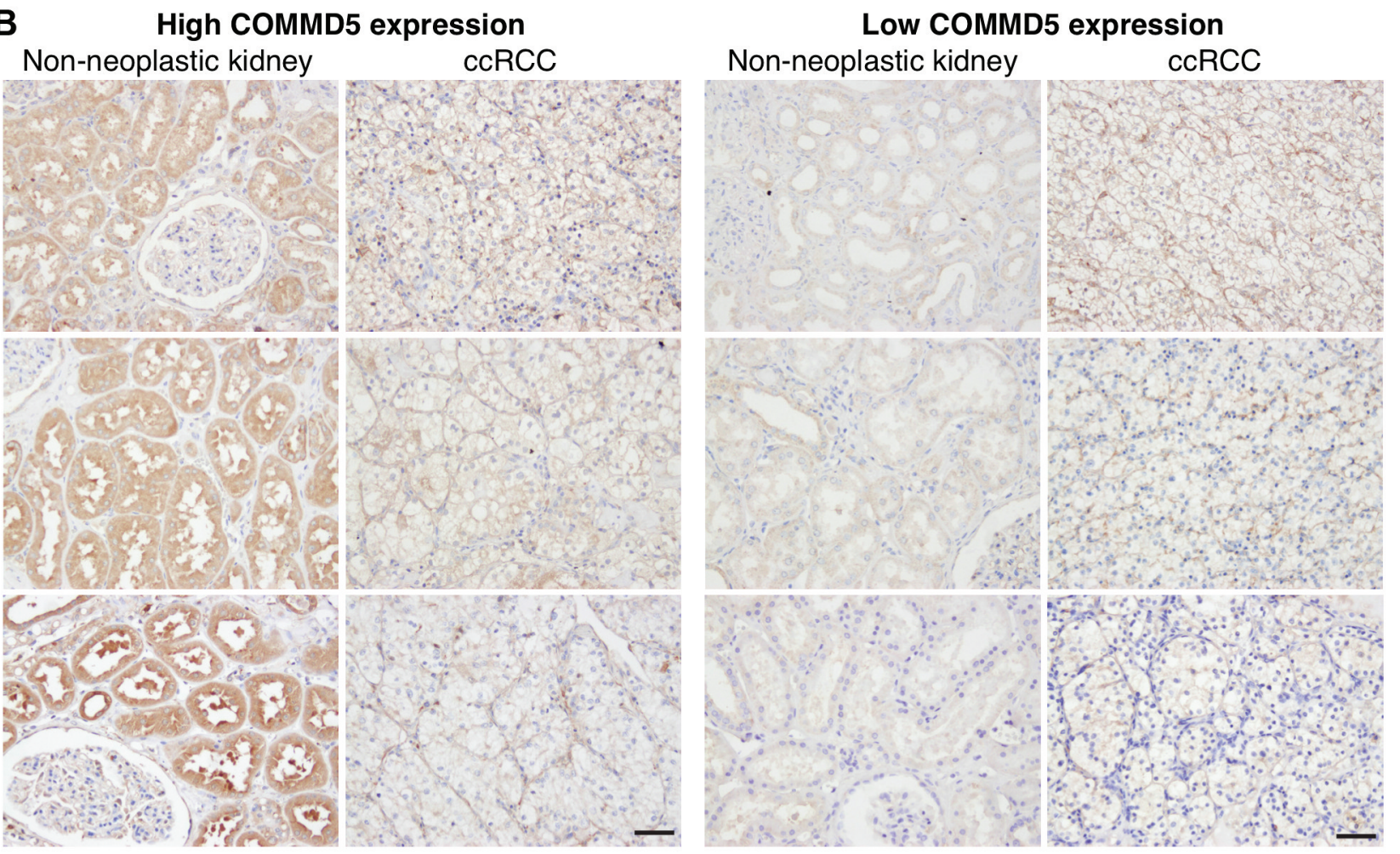

Figure 1. A: 5-Year metastasis and recurrence-free survival curves of patients with clear-cell renal cell carcinoma (ccRCC) according to tumor size (left) and expression of copper metabolism MURR1 domain-containing 5 (COMMD5) in non-neoplastic renal tubules. Small tumor size ( $\leq 40$ mm), and high COMMD5 level were associated with significantly better prognosis. B: Representative immunohistochemical staining of COMMD5 was performed on sections that included not only ccRCC but also normal kidney tissue adjacent to tumor. Examples of high (left) and low (right) expression of COMMD5 in non-neoplastic kidney tissues the adjacent to ccRCC. Scale bar=100 $\mu \mathrm{m}$.

1 cells (Figure 5B). When the suspensions of WT-RCC23 cells in the serum-free supernatant from COMMD5- or NeoTCMK-1 cells were placed on top of the basement membrane matrix, we noted after $24 \mathrm{~h}$ that the number of invading cells arising from incubation in the supernatant from COMMD5-TCMK-1 cells were significantly reduced compared with cells incubated in the supernatant from NeoTCMK-1 (Figure 5C), suggesting that secreted COMMD5 inhibits tumor cell invasion. As expected, supernatant from
COMMD5-TCMK-1 cells inhibited sphere formation compared with that from Neo-TCMK-1 cells 14 days after culture (Figure 5D).

\section{Discussion}

The incidence of kidney cancer is on the rise globally, and $\mathrm{RCC}$ remains one of the most lethal urological malignancies, despite improvements in its diagnosis and management (12, 


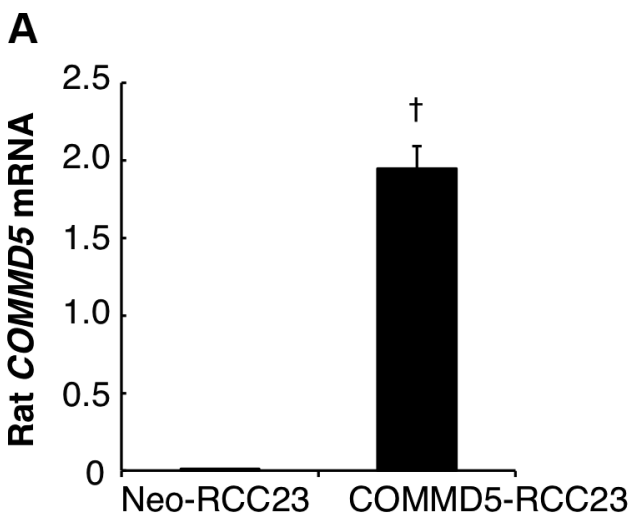

B
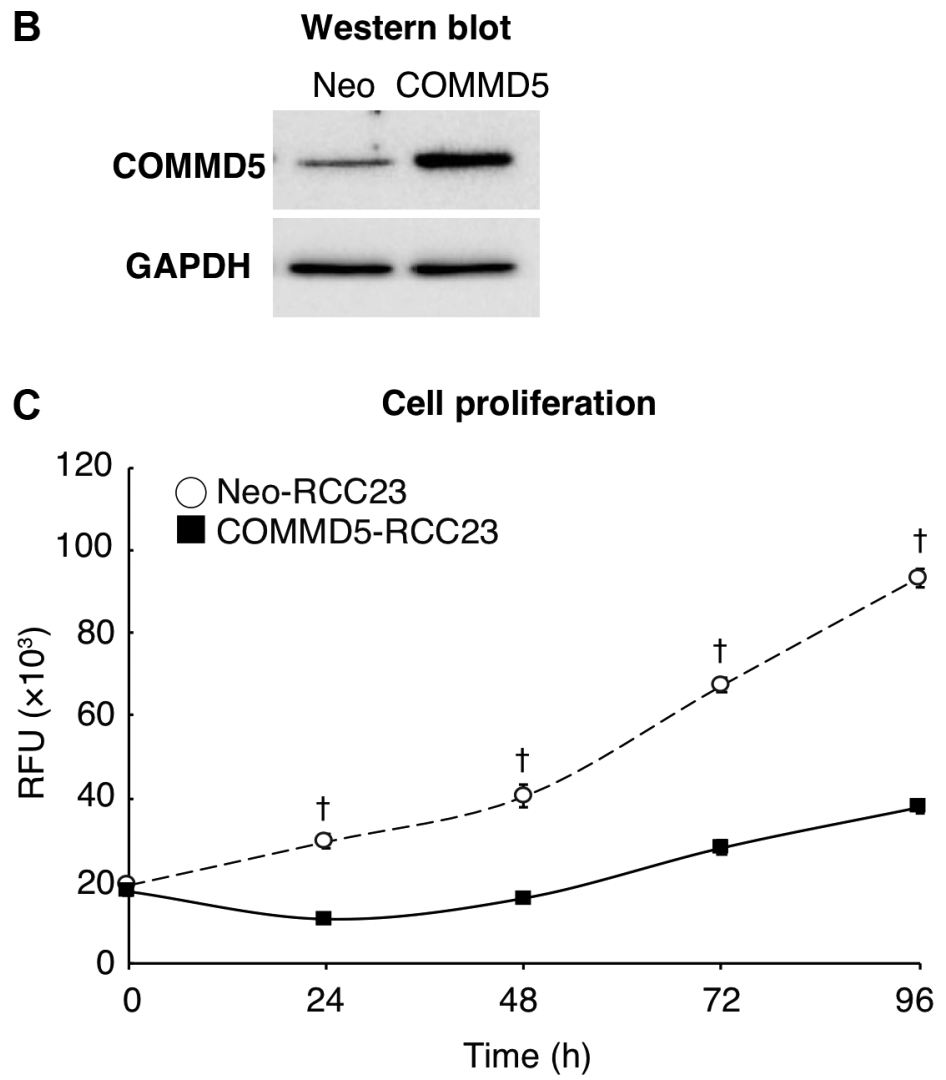

Figure 2. Copper metabolism MURR1 domain-containing 5 (COMMD5) overexpression inhibits proliferation of renal cell carcinoma (RCC) cells. A: Real-time polymerase chain reaction analysis of exogenous rat and endogenous human COMMD5 mRNA in RCC23 clones. In each experiment, expression levels were normalized to those of glyceraldehyde 3-phosphate dehydrogenase (GAPDH) mRNA. Exogenous COMMD5 was expressed only in COMMD5-RCC23 cells. The endogenous level of COMMD5 did not differ between Neo-control RCC23 cells and COMMD5-RCC23 cells. B: Total COMMD5 protein levels revealed by western blot were higher in COMMD5-RCC23 cells than in Neo-control cells. C: Growth curves of RCC23 clones. COMMD5-RCC23 cells proliferated less than Neo-RCC23 control cells. Values are the mean \pm standard error of the mean (n=6). †Significantly different at $p<0.0001$.

21). Moreover, RCC incidence rates are higher in rapidly developing countries, and this may be partly attributable to increases in the prevalence of obesity, hypertension and chronic kidney disease $(12,21,22)$. Hypertension is not only a well-established risk factor for the progression of renal failure but also increases the risk for RCC (23-28). We initially identified that COMMD5 was more highly expressed in the kidneys of hypertensive rats and facilitated repair of RTs after ischemic reperfusion injury $(2,4)$. Here we found that COMMD5 expression was suppressed in ccRCC specimens compared with adjacent kidney tissues. Tumor size at the time of surgery is one of the prognostic predictors of ccRCC, and nephron-sparing treatments for patients with stage T1a (40 mm or smaller lesions) are effective for preserving renal function $(29,30)$. Our univariate analysis showed that tumor size was a significant risk factor for metastasis and recurrence within 5 years after surgery, and the Kaplan-Meier plot indicated that a tumor size above 40 mm was significantly associated with poor metastasis- and recurrence-free survival. Despite the small sample size, our multivariate analysis showed that low COMMD5 expression in normal RTs was an independent risk factor for large tumor size compared with its high expression. Moreover, low COMMD5 expression was also a predictor for metastasis and recurrence in the univariate analysis. Based on the above findings, COMMD5 expression in the kidney might be effective in preventing tumorigenesis in ccRCC. Additional studies with an increased number of participants should be able to confirm or clarify the prognostic significance of the loss of COMMD5 in relation to ccRCC recurrence.

We previously reported that COMMD5 overexpression in murine renal cancer cells inhibited tumor growth and vascularization in a homograft tumor model (16). In the present study, we observed that both intracellular and secreted COMMD5 inhibited proliferation of human RCC23 cells, whereas loss of COMMD5 stimulated RCC23 cell 

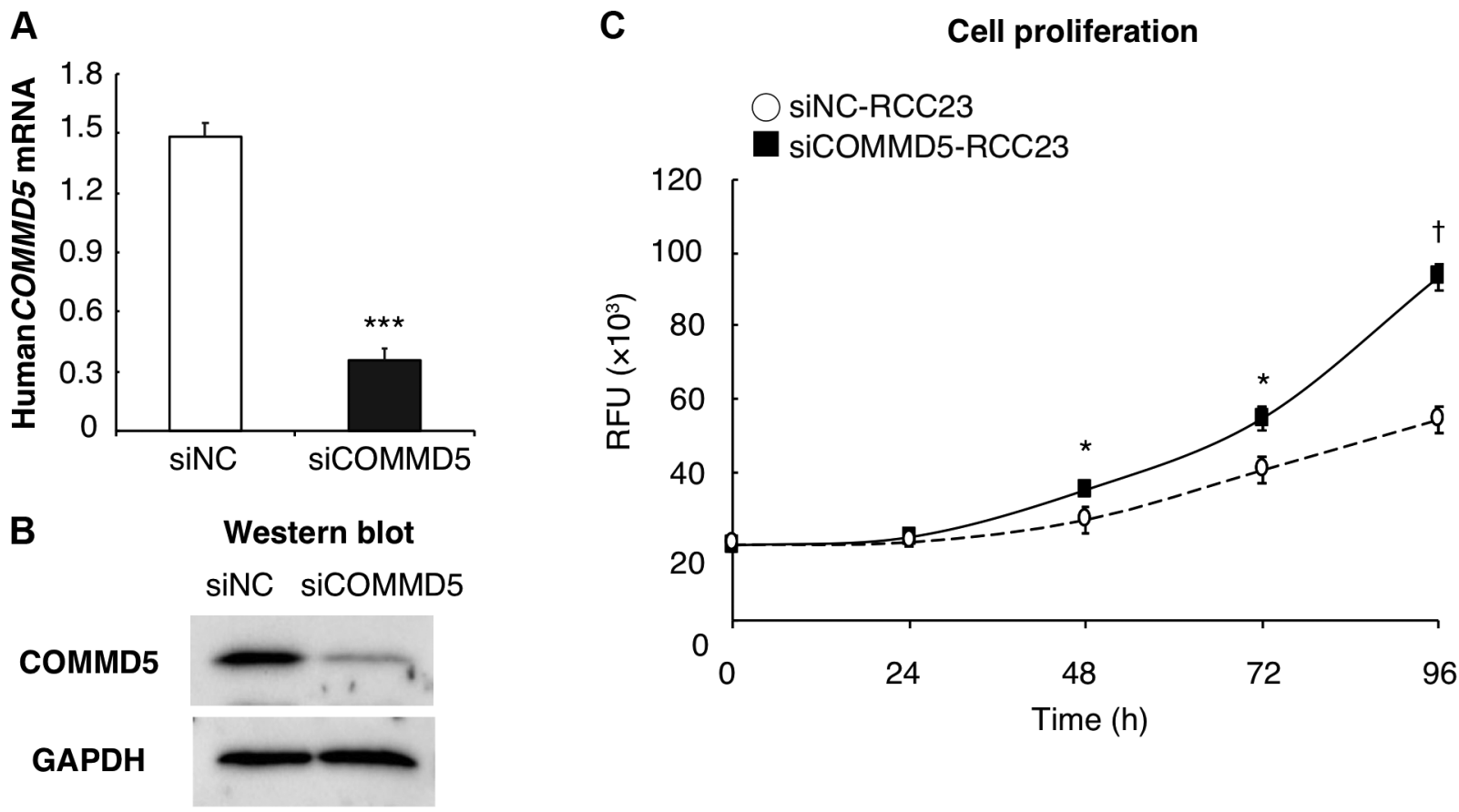

Figure 3. Loss of expression of copper metabolism MURR1 domain-containing 5 (COMMD5) facilitates renal cell carcinoma (RCC) cell proliferation. A: Real-time polymerase chain reaction analysis of endogenous human COMMD5 mRNA in RCC23 clones with the treatment of nontarget siRNA (siNC) or siRNA targeting COMMD5 sequence (siCOMMD5). Expression levels were normalized to those of glyceraldehyde 3phosphate dehydrogenase (GAPDH) mRNA levels. siCOMMD5 treatment reduced the mRNA level of COMMD5 by $80 \%$ compared with siNC-RCC23 control cells. B: COMMD5 protein levels revealed by western blot were lower in siCOMMD5-RCC23 cells than in siNC-RCC23 cells. C: Growth curves of RCC23 cells under treatment with siNC or siCOMMD5. siCOMMD5-RCC23 cells proliferated more than siNC-RCC23 cells. Values are the mean \pm standard error of the mean ( $n=6)$. Significantly different at: ${ }^{*} p<0.05, * * * p<0.0005$ and ${ }^{\dagger} p<0.0001$.

proliferation. We have reported that mechanistically, COMMD5 binds to endosomal RAS oncogene family member RAB5A and mediates epidermal growth factor receptor transport to sorting endosomes via the organization of actin and microtubule networks through RhoA signaling (31). Consequently, COMMD5 promotes de-phosphorylation of the erb-b2 receptor tyrosine kinase family, thus inactivating the phosphatidylinositol 3-kinase/protein kinase B signaling pathways which facilitate tumor cell proliferation (16).

RCC is the most common subtype of kidney cancer, and advanced RCC and metastatic RCC are resistant to conventional therapies, including chemotherapy and radiotherapy $(32,33)$. It was recently hypothesized that CSCs mediate resistance to most therapies, facilitate tumor metastasis and recurrence due to their ability for self-renew, and differentiate into heterogeneous cell types of the original tumor $(7,10,34,35)$. Renal CSCs have been identified and isolated from human RCCs by a specific marker of mesenchymal stem cells (36). CD $105^{+}$cells derived from RCCs were enriched in tumor-initiating stem cells that expressed stem cell markers. Renal CSCs have been frequently identified by use of stem cell markers such as
CD133 and CD44 $(19,37)$ and by use of several functional methods including the sphere-formation assay and ALDH activity (15, 38-40). However, there are contradicting reports on CSC markers and functional analysis. For example, CD133 expression was not restricted to CSCs of metastatic colon cancer (41), and side-population cells of gastrointestinal cancer were not enriched in CSCs (42). We examined the protein expression of several CSC markers in RCC23 cells, however, CD $44^{+}$or $\mathrm{CD} 133^{+}$RCC23 cells were not detected by flow cytometry. We therefore performed several types of functional analysis to assess the effects of COMMD5 on CSC-like properties of cells in italics studies. COMMD5 reduced the RCC23 cell population with high ALDH1 enzyme activity. High ALDH1 activity was shown to result in higher tumorigenicity and greater sphere-forming ability and invasive capability in several cancer types (43). As expected, our study showed COMMD5 reduced the invasiveness and sphere-forming ability of RCC23 cells. In addition, COMMD5 secreted from RT cells also reduced their CSC-like properties.

This study investigated the relationship between COMMD5 and tumor progression in patients with ccRCC. 
A

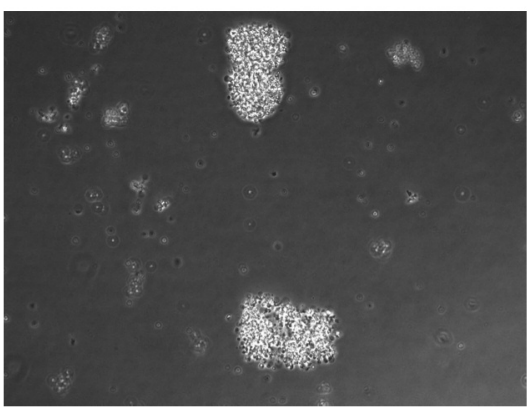

B
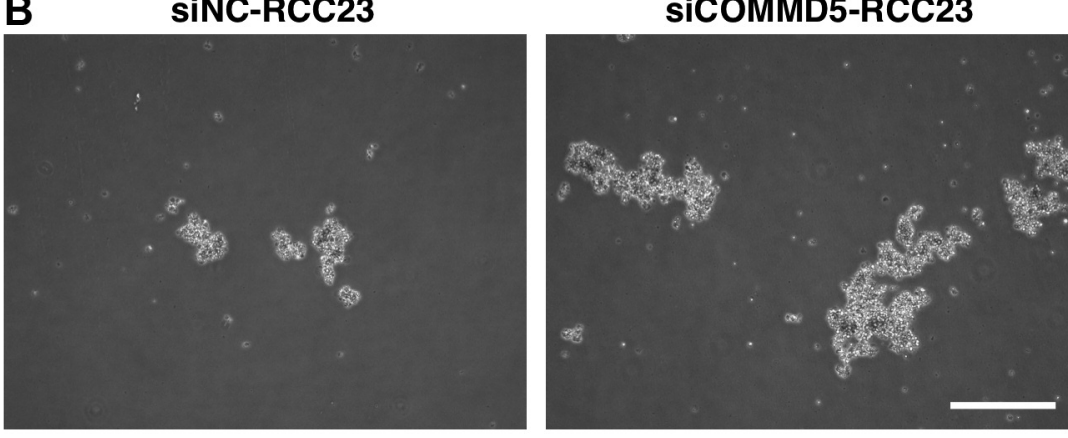

C

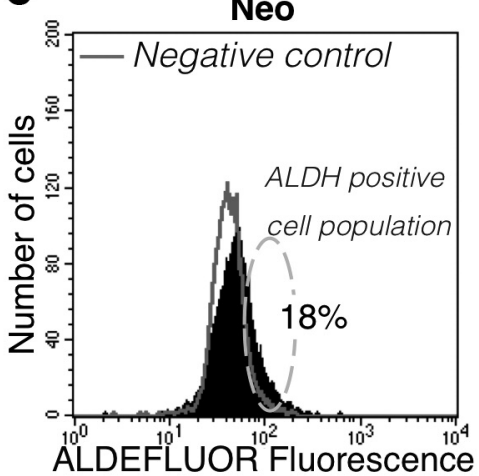

COMMD5-RCC23

siCOMMD5-RCC23

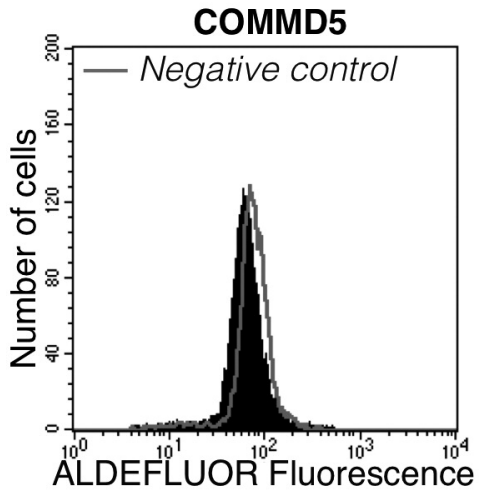

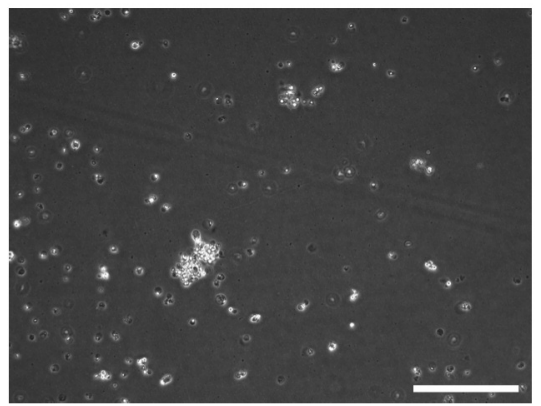

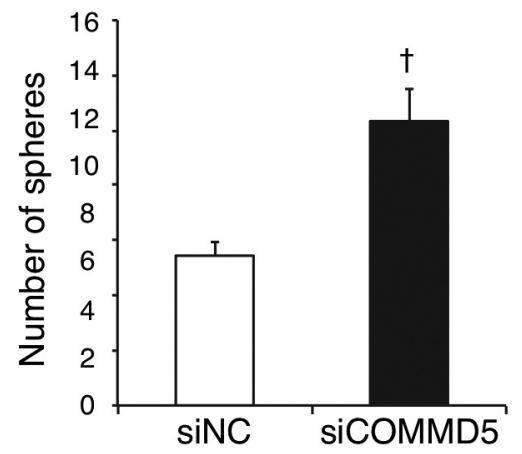

D
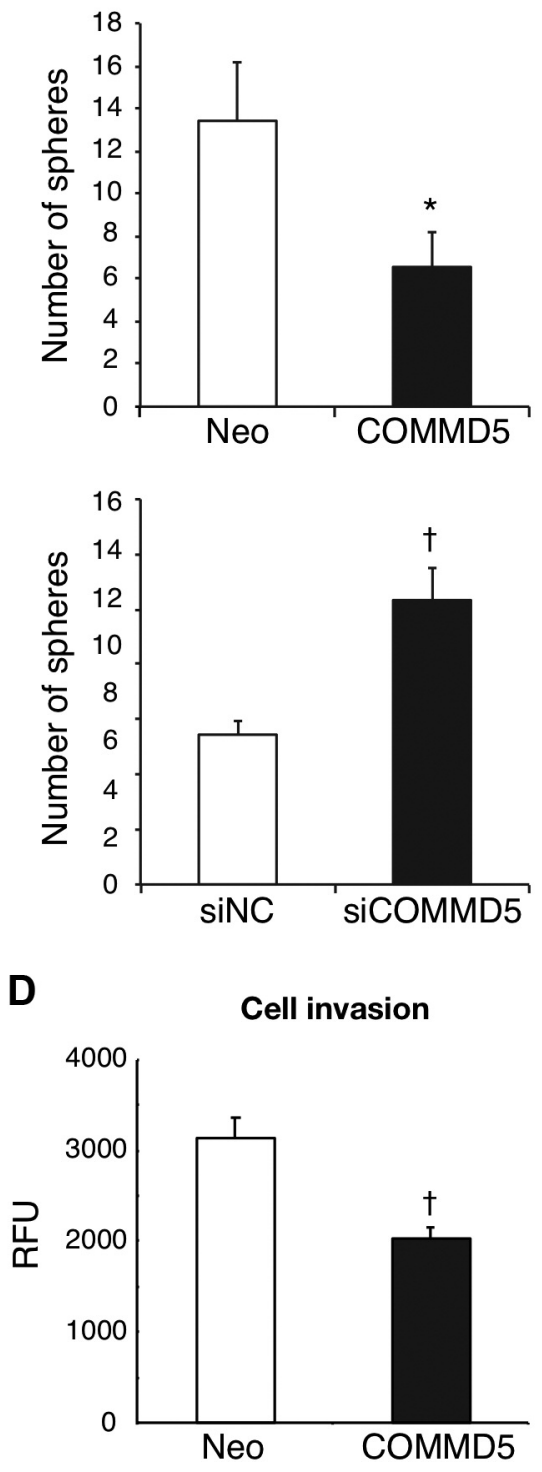

Figure 4. Copper metabolism MURR1 domain-containing 5 (COMMD5) inhibited cancer stemness of renal cell carcinoma (RCC) cells. Neo- and COMMD5-RCC23 cells (A) and cells transfected with non-target siRNA (siNC) or siRNA targeting COMMD5 sequence (siCOMMD5) (B) were incubated in a floating condition. Cell spheres of diameter greater than $500 \mu \mathrm{m}$ were counted after 14 days. COMMD5 significantly inhibited sphere formation by RCC23 cells. Scale bar=500 $\mathrm{m}$. C: ALDEFLOUOR assay of Neo- and COMMD5-RCC23 cells. The presence of aldehyde dehydrogenase (ALDH)-positive cells was indicated by a shift to the right compared with the negative control (gray line) Neo-RCC23 cells. Absence of ALDH activity was indicated by no shift to the right in COMMD5-RCC23 cells. Diethylaminobenzaldehyde, an ALDH-specific inhibitor, was used as the negative control. D: Cells were plated onto a basement membrane matrix and allowed to invade. After 24 h, cells that had invaded through the matrix were quantified, showing that COMMD5 overexpression inhibited invasion by RCC23 cells. Values are the mean \pm standard error of the mean ( $n=6)$. Significantly different at: $* p<0.05$ and ${ }^{\dagger} p<0.01$.

The results suggest that loss of COMMD5 in normal RTs might result in greater tumorigenicity, thus leading to poor clinical outcome. We also found that not only intracellular COMMD5, but also COMMD5 secreted from RT cells reduced malignant behaviors such as proliferation, invasion and sphere formation by RCC cells. Our results show that
COMMD5 might represent a promising therapeutic target for malignant RCC to improve patient outcomes.

\section{Conflicts of Interest}

The Authors declare no conflicts of interest. 

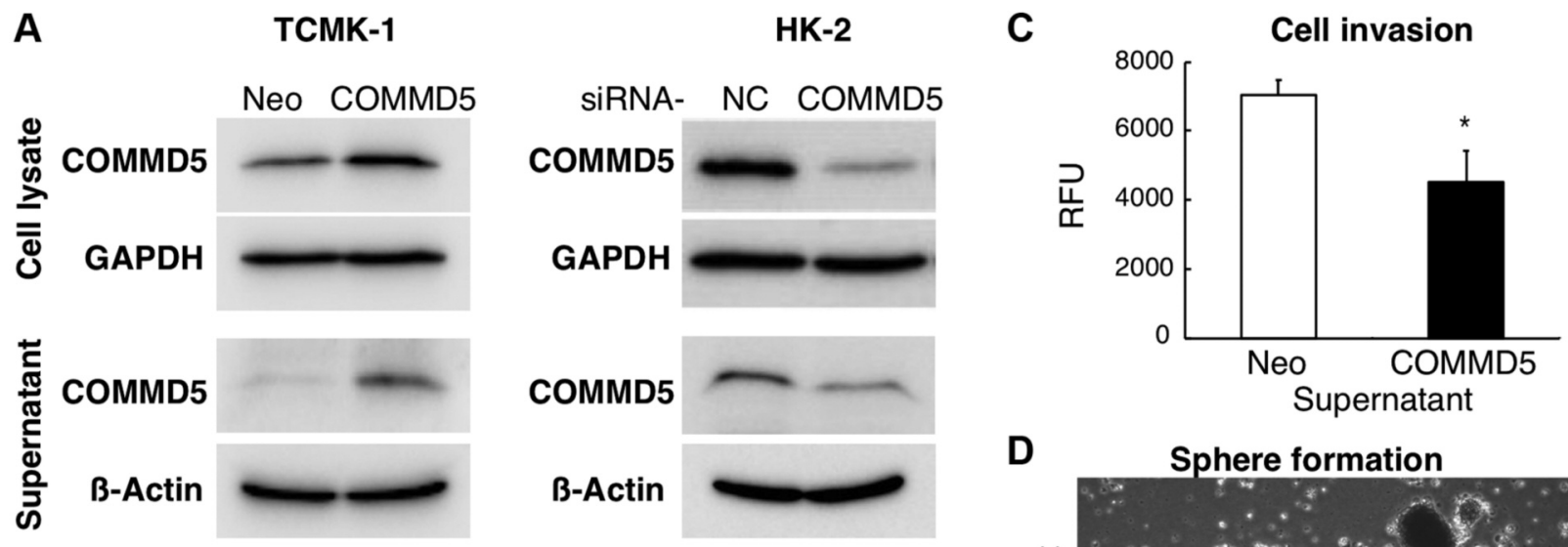

B

WT-RCC23 cell proliferation
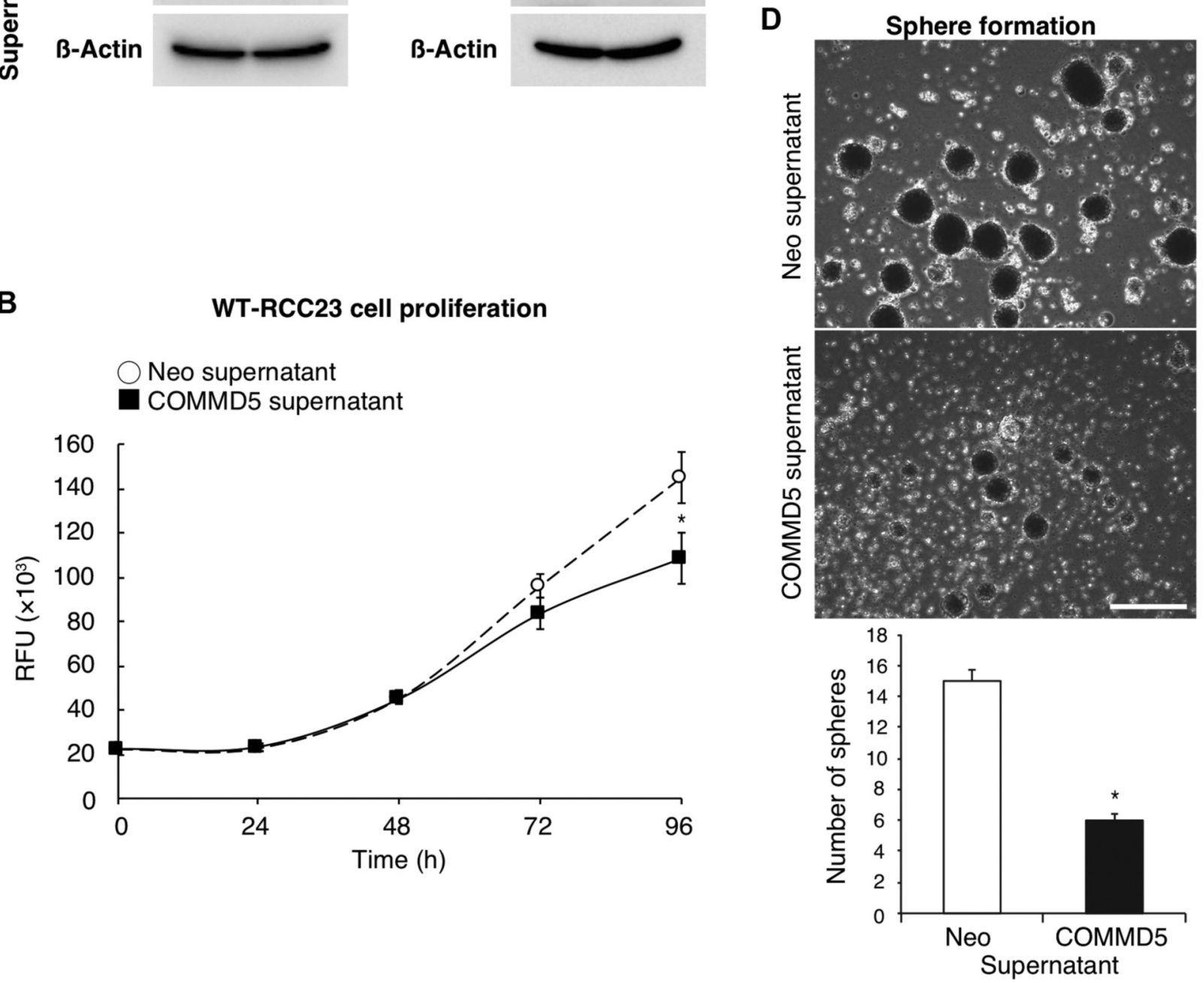

Figure 5. Secreted copper metabolism MURR1 domain-containing 5 (COMMD5) inhibited stemness of renal cell carcinoma (RCC) cells. COMMD5 was secreted from renal tubular cells. A: Total COMMD5 protein levels in cell lysates (upper panel) and acetone-precipitated supernatants of culture media (lower panel) from mouse kidney epithelial cells. Left: As revealed by western blot, COMMD5 protein levels were higher in COMMD5TCMK-1 cells than in Neo-TCMK-1 cells. Right: COMMD5 protein levels in cell lysates and their supernatants from human renal tubular epithelial cells. Protein levels in HK-2 cells after treatment with non-target siRNA (siNC) or siRNA targeting COMMD5 sequence (siCOMMD5) were lower in siCOMMD5-HK-2 cells than in siNC-HK-2 control cells. COMMD5 protein from siCOMMD5-HK-2 cells was secreted in much smaller amounts than conventional COMMD5. B: Growth curve of WT-RCC23 cells incubated with the supernatant from Neo-TCMK-1 cells or COMMD5-TCMK1 cells. Cell proliferation was inhibited in wild-type (WT)-RCC23 cells incubated with the supernatant from COMMD5-TCMK-1 cells compared with cells incubated with the supernatant from Neo-TCMK-1 cells. C: WT-RCC23 cells were plated onto a basement membrane matrix and incubated in supernatant from Neo- or COMMD5-TCMK-1 cells. After $24 \mathrm{~h}$, cells that had invaded through the matrix were quantified, showing that secreted COMMD5 inhibited invasion by WT-RCC23 cells. D: WT-RCC23 cells were incubated with supernatant from Neo- or COMMD5-TCMK-1 cells for 14 days. Cell spheres of diameter greater than $500 \mu \mathrm{m}$ were counted under the floating condition. Secreted COMMD5 significantly inhibited sphere formation by WT-RCC23 cells. Scale bar $=500 \mu \mathrm{m}$. Values are the mean \pm standard error of the mean $(n=6)$. *Significantly different at $p<0.05$. 


\section{Authors' Contributions}

Conception and design: Jin Ikeda and Hiroyuki Matsuda. Experiments, Data analysis and Interpretation: Jin Ikeda, Maiko Ogasawara, Hiroyuki Matsuda, and Morito Endo. Resources: Kenya Yamaguchi, Satoru Takahashi, Noboru Fukuda, Yoshikazu Masuhiro, Pavel Hamet, and Johanne Tremblay. Article Writing: All Authors. Final approval of article: All Authors.

\section{Acknowledgements}

The Authors are grateful to Suzanne Cossette for her expert technical assistance and advice. This work was supported in part by a Grant-in-Aid for Scientific Research from JSPS KAKENHI (18K08226 to H.M.) and a Nihon University Multidisciplinary Research Grant for 2018 (18-013 to H.M. and Y.M.).

\section{References}

1 Maine GN and Burstein E: COMMD proteins: COMMing to the scene. Cell Mol Life Sci 64(15): 1997-2005, 2007. PMID: 17497243. DOI: 10.1007/s00018-007-7078-y

2 Solban N, Jia HP, Richard S, Tremblay S, Devlin AM, Peng J, Gossard F, Guo DF, Morel G, Hamet P, Lewanczuk R and Tremblay J: HCaRG, a novel calcium-regulated gene coding for a nuclear protein, is potentially involved in the regulation of cell proliferation. J Biol Chem 275(41): 32234-32243, 2000. PMID: 10918053. DOI: $10.1074 /$ jbc.M001352200

3 Devlin AM, Solban N, Tremblay S, Gutkowska J, Schürch W, Orlov SN, Lewanczuk R, Hamet P and Tremblay J: HCaRG is a novel regulator of renal epithelial cell growth and differentiation causing G2M arrest. Am J Physiol Renal Physiol 284(4): F753F762, 2003. PMID: 12620924. DOI: 10.1152/ajprenal.00252.2002

4 Matsuda H, Lavoie JL, Gaboury L, Hamet P and Tremblay J: HCaRG accelerates tubular repair after ischemic kidney injury. J Am Soc Nephrol 22(11): 2077-2089, 2011. PMID: 21921141. DOI: 10.1681/ASN.2010121265

5 Erratum: Global cancer statistics 2018: GLOBOCAN estimates of incidence and mortality worldwide for 36 cancers in 185 countries. CA Cancer J Clin 70(4): 313, 2020. PMID: 32767693. DOI: $10.3322 /$ caac. 21609

6 Toledo-Guzmán ME, Bigoni-Ordóñez GD, Ibáñez Hernández M and Ortiz-Sánchez E: Cancer stem cell impact on clinical oncology. World J Stem Cells 10(12): 183-195, 2018. PMID: 30613312. DOI: 10.4252/wjsc.v10.i12.183

7 Dean M, Fojo $\mathrm{T}$ and Bates S: Tumour stem cells and drug resistance. Nat Rev Cancer 5(4): 275-284, 2005. PMID: 15803154. DOI: $10.1038 / \mathrm{nrc} 1590$

8 Bighetti-Trevisan RL, Sousa LO, Castilho RM and Almeida LO: Cancer stem cells: Powerful targets to improve current anticancer therapeutics. Stem Cells Int 2019: 9618065, 2019. PMID: 31781251. DOI: 10.1155/2019/9618065

9 Reya T, Morrison SJ, Clarke MF and Weissman IL: Stem cells, cancer and cancer stem cells. Nature 414(6859): 105-111, 2001. PMID: 11689955 . DOI: $10.1038 / 35102167$

10 Visvader JE and Lindeman GJ: Cancer stem cells in solid tumours: accumulating evidence and unresolved questions. Nat Rev Cancer 8(10): 755-768, 2008. PMID: 18784658. DOI: $10.1038 /$ nrc2499
11 Turajlic S, Swanton C and Boshoff C: Kidney cancer: The next decade. J Exp Med 215(10): 2477-2479, 2018. PMID: 30217855. DOI: $10.1084 /$ jem.20181617

12 Rossi SH, Klatte T, Usher-Smith $\mathrm{J}$ and Stewart GD: Epidemiology and screening for renal cancer. World J Urol 36(9): 1341-1353, 2018. PMID: 29610964. DOI: 10.1007/ s00345-018-2286-7

13 Coppin C, Kollmannsberger C, Le L, Porzsolt F and Wilt TJ: Targeted therapy for advanced renal cell cancer (RCC): a Cochrane systematic review of published randomised trials. BJU Int 108(10): 1556-1563, 2011. PMID: 21952069. DOI: 10.1111/j.1464-410X.2011.10629.x

14 Khan MI, Czarnecka AM, Helbrecht I, Bartnik E, Lian F and Szczylik C: Current approaches in identification and isolation of human renal cell carcinoma cancer stem cells. Stem Cell Res Ther 6: 178, 2015. PMID: 26377541. DOI: 10.1186/s13287-0150177-z

15 Huang B, Huang YJ, Yao ZJ, Chen X, Guo SJ, Mao XP, Wang $\mathrm{DH}$, Chen JX and Qiu SP: Cancer stem cell-like side population cells in clear cell renal cell carcinoma cell line 769P. PLoS One 8(7): e68293, 2013. PMID: 23874578. DOI: 10.1371/journal. pone. 0068293

16 Matsuda H, Campion CG, Fujiwara K, Ikeda J, Cossette S, Verissimo T, Ogasawara M, Gaboury L, Saito K, Yamaguchi K, Takahashi S, Endo M, Fukuda N, Soma M, Hamet P and Tremblay J: HCaRG/COMMD5 inhibits ErbB receptor-driven renal cell carcinoma. Oncotarget 8(41): 69559-69576, 2017. PMID: 29050225. DOI: 10.18632/oncotarget.18012

17 Vandesompele J, De Preter K, Pattyn F, Poppe B, Van Roy N, De Paepe A and Speleman F: Accurate normalization of realtime quantitative RT-PCR data by geometric averaging of multiple internal control genes. Genome Biol 3(7): RESEARCH0034, 2002. PMID: 12184808. DOI: 10.1186/gb2002-3-7-research0034

18 Ginestier C, Hur MH, Charafe-Jauffret E, Monville F, Dutcher J, Brown M, Jacquemier J, Viens P, Kleer CG, Liu S, Schott A, Hayes D, Birnbaum D, Wicha MS and Dontu G: ALDH1 is a marker of normal and malignant human mammary stem cells and a predictor of poor clinical outcome. Cell Stem Cell 1(5): 555567, 2007. PMID: 18371393. DOI: 10.1016/j.stem.2007.08.014

19 Gassenmaier M, Chen D, Buchner A, Henkel L, Schiemann M, Mack B, Schendel DJ, Zimmermann W and Pohla H: CXC chemokine receptor 4 is essential for maintenance of renal cell carcinoma-initiating cells and predicts metastasis. Stem Cells 31(8): 1467-1476, 2013. PMID: 23630186. DOI: 10.1002/ stem.1407

20 Mele L, Liccardo D and Tirino V: Evaluation and isolation of cancer stem cells using ALDH activity assay. Methods Mol Biol 1692: 43-48, 2018. PMID: 28986885. DOI: 10.1007/978-14939-7401-6_4

21 Capitanio U, Bensalah K, Bex A, Boorjian SA, Bray F, Coleman J, Gore JL, Sun M, Wood C and Russo P: Epidemiology of renal cell carcinoma. Eur Urol 75(1): 74-84, 2019. PMID: 30243799. DOI: $10.1016 /$ j.eururo.2018.08.036

22 Chow WH, Dong LM and Devesa SS: Epidemiology and risk factors for kidney cancer. Nat Rev Urol 7(5): 245-257, 2010. PMID: 20448658. DOI: 10.1038/nrurol.2010.46

23 Hamet P: Cancer and hypertension: a potential for crosstalk? J Hypertens 15(12 Pt 2): 1573-1577, 1997. PMID: 9488208. DOI: 10.1097/00004872-199715120-00058 
24 Whitworth JA: Progression of renal failure - the role of hypertension. Ann Acad Med Singap 34(1): 8-15, 2005. PMID: 15726214.

25 Choi MY, Jee SH, Sull JW and Nam CM: The effect of hypertension on the risk for kidney cancer in Korean men. Kidney Int 67(2): 647-652, 2005. PMID: 15673312. DOI: 10.1111/j.1523-1755.2005.67137.x

26 Setiawan VW, Stram DO, Nomura AM, Kolonel LN and Henderson BE: Risk factors for renal cell cancer: the multiethnic cohort. Am J Epidemiol 166(8): 932-940, 2007. PMID: 17656615. DOI: 10.1093/aje/kwm170

27 Wang G, Hou J, Ma L, Xie J, Yin J, Xu D, Chang W, Tan X, Su T, Zhang $\mathrm{H}$ and Cao G: Risk factor for clear cell renal cell carcinoma in Chinese population: a case-control study. Cancer Epidemiol 36(2): 177-182, 2012. PMID: 22000673. DOI: 10.1016/j.canep.2011.09.006

28 Matsuda H, Hamet P and Tremblay J: Hypertension-related, calcium-regulated gene (HCaRG/COMMD5) and kidney diseases: HCaRG accelerates tubular repair. J Nephrol 27(4): 351-360, 2014. PMID: 24515317. DOI: 10.1007/s40620-0140054-3

29 Rini BI, Rathmell WK and Godley P: Renal cell carcinoma. Curr Opin Oncol 20(3): 300-306, 2008. PMID: 18391630. DOI: 10.1097/CCO.0b013e3282f9782b

30 Lucas SM, Stern JM, Adibi M, Zeltser IS, Cadeddu JA and Raj $\mathrm{GV}$ : Renal function outcomes in patients treated for renal masses smaller than $4 \mathrm{~cm}$ by ablative and extirpative techniques. J Urol 179(1): 75-9; discussion 79-80, 2008. PMID: 17997440. DOI: 10.1016/j.juro.2007.08.156

31 Campion CG, Zaoui K, Verissimo T, Cossette S, Matsuda H, Solban N, Hamet P and Tremblay J: COMMD5/HCaRG hooks endosomes on cytoskeleton and coordinates EGFR trafficking. Cell Rep 24(3): 670-684.e7, 2018. PMID: 30021164. DOI: $10.1016 /$ j.celrep.2018.06.056

32 Flanigan RC, Campbell SC, Clark JI and Picken MM: Metastatic renal cell carcinoma. Curr Treat Options Oncol 4(5): 385-390, 2003. PMID: 12941198. DOI: 10.1007/s11864-003-0039-2

33 Canda AE and Kirkali Z: Current management of renal cell carcinoma and targeted therapy. Urol J 3(1): 1-14, 2006. PMID: 17590846.

34 Phi LTH, Sari IN, Yang YG, Lee SH, Jun N, Kim KS, Lee YK and Kwon HY: Cancer stem cells (CSCs) in drug resistance and their therapeutic implications in cancer treatment. Stem Cells Int 2018: 5416923, 2018. PMID: 29681949. DOI: 10.1155/ $2018 / 5416923$

35 Adorno-Cruz V, Kibria G, Liu X, Doherty M, Junk DJ, Guan D, Hubert C, Venere M, Mulkearns-Hubert E, Sinyuk M, Alvarado A, Caplan AI, Rich J, Gerson SL, Lathia J and Liu H: Cancer stem cells: targeting the roots of cancer, seeds of metastasis, and sources of therapy resistance. Cancer Res 75(6): 924-929, 2015. PMID: 25604264. DOI: 10.1158/0008-5472.CAN-14-3225
36 Bussolati B, Bruno S, Grange C, Ferrando U and Camussi G: Identification of a tumor-initiating stem cell population in human renal carcinomas. FASEB J 22(10): 3696-3705, 2008. PMID: 18614581. DOI: 10.1096/fj.08-102590

37 Bruno S, Bussolati B, Grange C, Collino F, Graziano ME, Ferrando U and Camussi G: CD133+ renal progenitor cells contribute to tumor angiogenesis. Am J Pathol 169(6): 22232235, 2006. PMID: 17148683. DOI: 10.2353/ajpath. 2006.060498

38 Ueda K, Ogasawara S, Akiba J, Nakayama M, Todoroki K, Ueda K, Sanada S, Suekane S, Noguchi M, Matsuoka K and Yano H: Aldehyde dehydrogenase 1 identifies cells with cancer stem celllike properties in a human renal cell carcinoma cell line. PLoS One 8(10): e75463, 2013. PMID: 24116047. DOI: 10.1371/journal.pone.0075463

39 Chen DC, Liang YD, Peng L, Wang YZ, Ai CZ, Zhu XX, Yan YW, Saeed Y, Yu B, Huang J, Gao Y, Liu J, Jiang YZ, Liu M and Chen D: CBFA2T2 is associated with a cancer stem cell state in renal cell carcinoma. Cancer Cell Int 17: 103, 2017. PMID: 29162985. DOI: 10.1186/s12935-017-0473-Z

40 Wang L, Park P, La Marca F, Than KD and Lin CY: BMP-2 inhibits tumor-initiating ability in human renal cancer stem cells and induces bone formation. J Cancer Res Clin Oncol 141(6): 1013-1024, 2015. PMID: 25431339. DOI: 10.1007/s00432-0141883-0

41 Shmelkov SV, Butler JM, Hooper AT, Hormigo A, Kushner J, Milde T, St Clair R, Baljevic M, White I, Jin DK, Chadburn A, Murphy AJ, Valenzuela DM, Gale NW, Thurston G, Yancopoulos GD, D’Angelica M, Kemeny N, Lyden D and Rafii S: CD133 expression is not restricted to stem cells, and both CD133+ and CD133- metastatic colon cancer cells initiate tumors. J Clin Invest 118(6): 2111-2120, 2008. PMID: 18497886. DOI: $10.1172 / J C I 34401$

42 Burkert J, Otto WR and Wright NA: Side populations of gastrointestinal cancers are not enriched in stem cells. J Pathol 214(5): 564-573, 2008. PMID: 18266310. DOI: 10.1002/ path. 2307

43 Kuroda T, Hirohashi Y, Torigoe T, Yasuda K, Takahashi A, Asanuma H, Morita R, Mariya T, Asano T, Mizuuchi M, Saito $\mathrm{T}$ and Sato N: ALDH1-high ovarian cancer stem-like cells can be isolated from serous and clear cell adenocarcinoma cells, and ALDH1 high expression is associated with poor prognosis. PLoS One 8(6): e65158, 2013. PMID: 23762304. DOI: 10.1371/ journal.pone .0065158
Received April 22, 2021

Revised May 10, 2021

Accepted May 17, 2021 